\title{
Self-Growing RBF Neural Network Approach for Semantic Image Retrieval
}

\author{
Li Guizhi ${ }^{*}$ and Huang Hongbo
}

\author{
Computer Center, Beijing Information and Science and Technology University, Beijing, 100192, P.R. China
}

\begin{abstract}
Traditional methods of content-based image retrieval deal with the retrieval of images according to the similarity between them and the sample image in some low-level feature space such as color, shape and structure. But the relevant images satisfying user information need tend to have different distribution in the low-level feature space. In this case, the query image needs to be represented as multiple query images corresponding to the scattered relevant images. This paper proposes a new relevance feedback technique for semantic image retrieval which is based on the self-growing radial basis function (SGRBF) neural network. The approach can adaptively construct SGRBF neural network based on the users' feedbacks. Thus, hidden nodes of the SGRBF neural network can represent the distribution of the users' perceptual in the low-level feature space and bridge the semantic gap between low-level feature and high-level concept of the image content. The method is verified on a database of 1000 images and experimental results demonstrate that our method proposed in this paper is an effective method to promote semantic image retrieval performance.
\end{abstract}

Keywords: Relevance feedback, SGRBF neural network, semantic image retrieval.

\section{INTRODUCTION}

Content-based image retrieval (CBIR) has broad application prospects and has become a popular research fields. At present, the semantic gap between low-level image features and high-level image meanings existing in the CBIR systems greatly affects the systems' performance. Image retrieval based on semantics is a challenging problem due to the lack of mature technique mapping high-level semantic concepts to low-level feature descriptors.

Content-based image retrieval systems have introduced relevance feedback approaches to facilitate bridging the semantic gap between low-level feature descriptors and highlevel semantic concepts [1-6]. Relevance feedback is an human-computer interaction technique during the image retrieval process, which lets the user label the retrieved images as positive or negative. The systems reuse these labeled sample images to refine retrieval results by a machine learning approaches.

Adopting relevance feedback technique in CBIR systems have improved considerably performance of image retrieval as it integrates the user's evaluation on the retrieval results into the retrieval procedure, and the last results can better satisfy the user information need [7-11]. Traditional approaches of image retrieval return the images according to the similarity between them and the user query in the lowlevel feature space. However, the similarity of visual features representing the color, texture or shape information does not necessarily reflect the high-level semantics similarity because semantically relevant images tend to have several different clusters distributed in the low-level feature space. Traditional relevance feedback techniques are not suitable for handing this situation and thus affect the performance of CBIR.

This paper proposes a new relevance feedback approach using SGRBF neural network for semantic image retrieval. The approach can adaptively construct SGRBF neural network based on the users' relevance feedbacks. Thus, the neighboring hidden nodes of the SGRBF neural network can describe the distribution of the users' perceptual in the lowlevel feature space and bridge the semantic gap between lowlevel feature and high-level concept of the image content.

We organize the remainder of this paper as follows. Section II introduces the principle of radial basis function neural network. The use of self-growing radial basis function neural network is described in section III. The SGRBF neural network based semantic image retrieval technique is discussed in section 4. Section 5 presents our experimental results. At last, some conclusions of this paper are given in section 6 .

\section{RBF NETWORK ARCHITECTURE}

The architecture of a RBF neural network is depicted in Fig. (1). The role of the input layer is to transfer the input vector $x=\left[x_{1}, x_{2}, \mathrm{~L}, x_{n}\right]^{T}$ to the next hidden layer through scalar weights. The hidden nodes represent the RBF units which carry out the RBF computation over the input vector transferred from input layer and transfer the result to the 


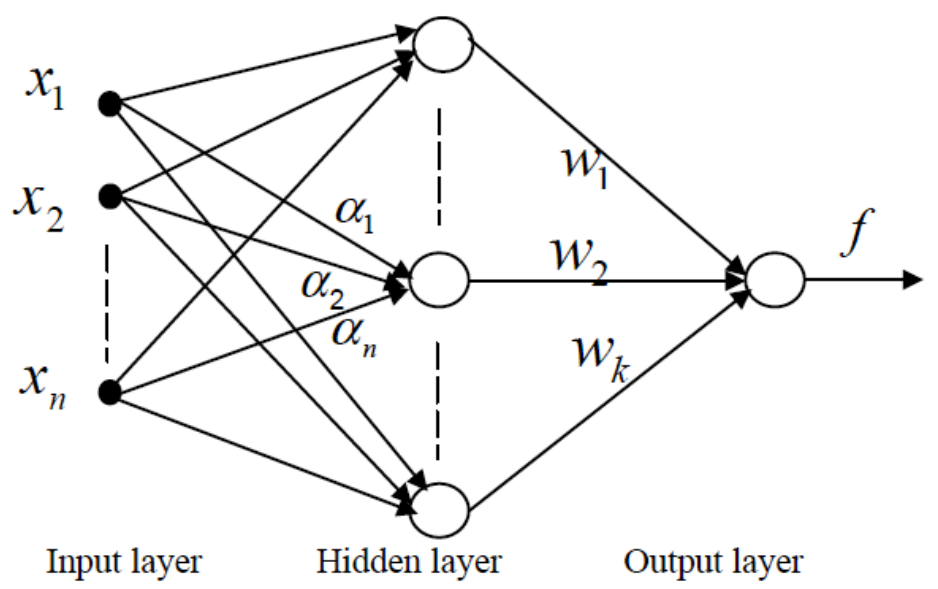

Fig. (1). The architecture of RBF neural network.

output layer. The output layer computes final result by linear superposition of the $k$ outputs from the RBF units. The result output by the RBF neural network can be represented as follows [12]:

$y=f(x)=\sum_{i=1}^{k} w_{i} \phi_{i}(x)$

where $f(x)$ is the final result for a input vector $x=\left[x_{1}, x_{2}, \cdots, x_{n}\right]^{T}, \phi_{i}(\cdot)$ gives the output of the $i$ th RBF unit, $w_{i}$ is the weight connecting the $i$ th RBF unit and the output node, and $k$ is the total number of RBF units .

The degree of similarity between the input vector and a RBF cluster center is described by a radial basis function. Usually the RBF neural network use Gaussian function to describe the distribution of the data clusters. As different components of the input vector may have different degrees of importance, the Gaussian function is defined as:

$\phi_{i}(x)=\exp \left(-\sum_{j=1}^{n} \alpha_{j}\left(x_{j}-u_{i j}\right)^{2} /\left(2 \sigma_{i}^{2}\right)\right)$

where the importance weight of different feature components is denoted by $\alpha_{j}, u_{i} \in R^{n}$ is the $i$ th RBF cluster's center and $\sigma_{i}$ represents its width, .

\section{THE SGRBF NEURAL NETWORK}

The number of the hidden nodes and their locations are important parameters affecting the performance of SGRBF neural network. To deal with this problem, the developed SGRBF neural network can automatically adjust the parameters of the RBF hidden nodes [12-14].

The learning procedure of self-growing RBF neural network can be described as follows: at first, there is no RBF node in the middle layer. Then the network chooses one sample randomly from input training sample as the first RBF unit center $u_{1}$. Next, the Euclidean distance between the center $u_{1}$ and the next training data $x$ is computed. If it is greater than the parameter $\varepsilon$, the network will create a new RBF unit $u_{2}$ centered at $x$, otherwise the value of $u_{1}$ is updated as:

$u_{1}=u_{1}+\eta\left\|x-u_{1}\right\|$

where $|| \mid$. represents the Euclidean distance and $0<\eta<1$ denotes the updating weight. This process continues until the remaining training samples are processed. At last, the clusters' number increases and the locations of cluster centers can be automatically adjusted with the learning procedure.

\section{SEMANTIC IMAGE RETRIEVAL BASED ON SGRBF}

Semantic image retrieval with relevance feedback based on SGRBF use the RBF unit centers produced by the neural network learning representing the query. Through continuous learning, The SGRBF neural network can gradually simulate real image semantics relevance distribution. The learning procedure starts with a sample image supplied by the user. Then the images in the databases are retrieved according to the distance between them and the sample image. These retrieved images are provided to the user for feedback. Those images closest to the sample image are labeled as positive by the user, while the remaining images are labeled as negative. At last, these labeled images are used as samples to train the RBF network and the parameters of RBF nodes are modified. In the next search, the system uses the re-adjusted RBF neural network to compute the semantic similarity of the image. The learning procedure continues until the user obtains the retrieval images he need.

Semantic image retrieval based on SGRBF neural network learning includes two steps. The first step is to use positive images labeled by the user to construct SGRBF neural network. The second step is to adjust the network parameters using the negative images. 


\subsection{RBF Network Self-Growth Technique}

The purpose of this paper is to use multiple queries to characterize the query image instead of modifying a single query. Positive images are utilized to construct the SGRBF neural network because they are the images the user expected. The RBF units are modified through the learning process. The algorithm can be described as follows:

1. Supposing that $\left\{u_{i}\right\}_{i=1}^{k}$ represent $k$ hidden nodes, which are created through previous learning procedure. In the next iteration, for each labeled relevant image $x^{\prime} \in R^{n}$, where $n$ denotes the input vector dimension, the system calculates its similarity with each RBF unit $\left\|x^{\prime}-u_{i}\right\|$ for $i=1, \cdots, k$.

2. The system searches the best-matching RBF unit according to the following condition:

$\left\|x^{\prime}-u_{j}\right\| \leq\left\|x^{\prime}-u_{i}\right\|, \forall i \neq j$

3. Then the $\left\|x^{\prime}-u_{j}\right\|$ is compared with the threshold value $\mathcal{\varepsilon}$. If it is greate

4. $\mathrm{r}$ than the parameter, a new RBF unit $u_{k+1}$ is added to the network centered at the image $x^{\prime}$. Otherwise the relevant image $x^{\prime}$ is added to the $j$ th unit and the RBF unit center $u_{j}$ is updated by:

$u_{j}=u_{j}+\eta\left\|x^{\prime}-u_{j}\right\|$

5. The above steps are repeated to deal with the rest relevant image until all of the positive images labeled by the user are processed.

\subsection{SGRBF Neural Network Learning}

Because the irrelevant images $x^{\prime \prime} \in R^{n}$ labeled by the user are similar to the sample image based on the previous similarity calculation, theses images are employed to adjust the network parameters. The anti-reinforced learning rule is adopted on the RBF units, which make them move away from irrelevant data. Let $x^{\prime \prime}$ be the nearest irrelevant sample to the unit $u_{i}$. The cluster center is adjusted as follows:

$u_{i}=u_{i}-\eta_{2}\left(x^{\prime \prime}-u_{i}\right)$

where $\eta_{2}$ denotes the learning parameter.

For an image feature vector $x$ from the database, the overall output $f(x)$ functioning as a similarity measurement is calculated through the linear superposition of all the outputs from each RBF unit. The images with higher values are more likely to be the retrieval target satisfied by the user.

\subsection{Determination of SGRBF Neural Network Parame- ters}

Different feature components may have different degrees of importance in relevance evaluation. So various feature variables should be given a weight value to denote their importance. Where $\alpha_{j}$ in equation (2) are the importance weight of different feature variables which are obtained through the variance computation from the relevant images as follows:

$$
\alpha_{j}= \begin{cases}1 & \xi_{j}=0 \\ 1 / \xi_{j} & \xi_{j}>0\end{cases}
$$

where $\xi_{j}$ represents the $j$ th feature component variance in the relevant samples. If a feature component is important in relevance evaluation, all relevant images should have a very similar value on this feature component. Accordingly the variance value on this feature component is small.

Not all relevant images are equally important in satisfying the user's image perceptual. At the user's evaluation step, the user gives a weight value $v_{i}$ for each positive image $x_{i}$. During the SGRBF neural network training, if $x_{i}$ is added to the $j$ th $\mathrm{RBF}$ unit as the $k t h$ point, then the weight value of $j$ th cluster is:

$m_{j}=\sum_{i=1}^{k} v_{i}$

Then the normalized weight value of the $j$ th $\mathrm{RBF}$ unit is given by:

$w_{j}=m_{j} / \sum_{i=1}^{k} m_{i}$

At last, the cluster width is defined as:

$\sigma_{i}=\delta \cdot \min _{j}|| u_{i}-u_{j} \| j=1,2, \ldots, k$

Where $\delta$ is a parameter which indicates the overlapping of different clusters.

\section{EXPERIMENTAL RESULTS}

The experiment is carried on the image database containing 1000 color images which are divided into 10 different classes. In the experiment, color and texture visual features are used. We use color histogram and color moments as the color features, and Gabor wavelet transform as the texture features.

Total 10 images from each image class are selected as initial queries in the experiment. The system retrieves $20 \mathrm{im}-$ ages for user feedback at each iteration. Total 5 feedback iterations are performed to verify the image retrieval quality. 


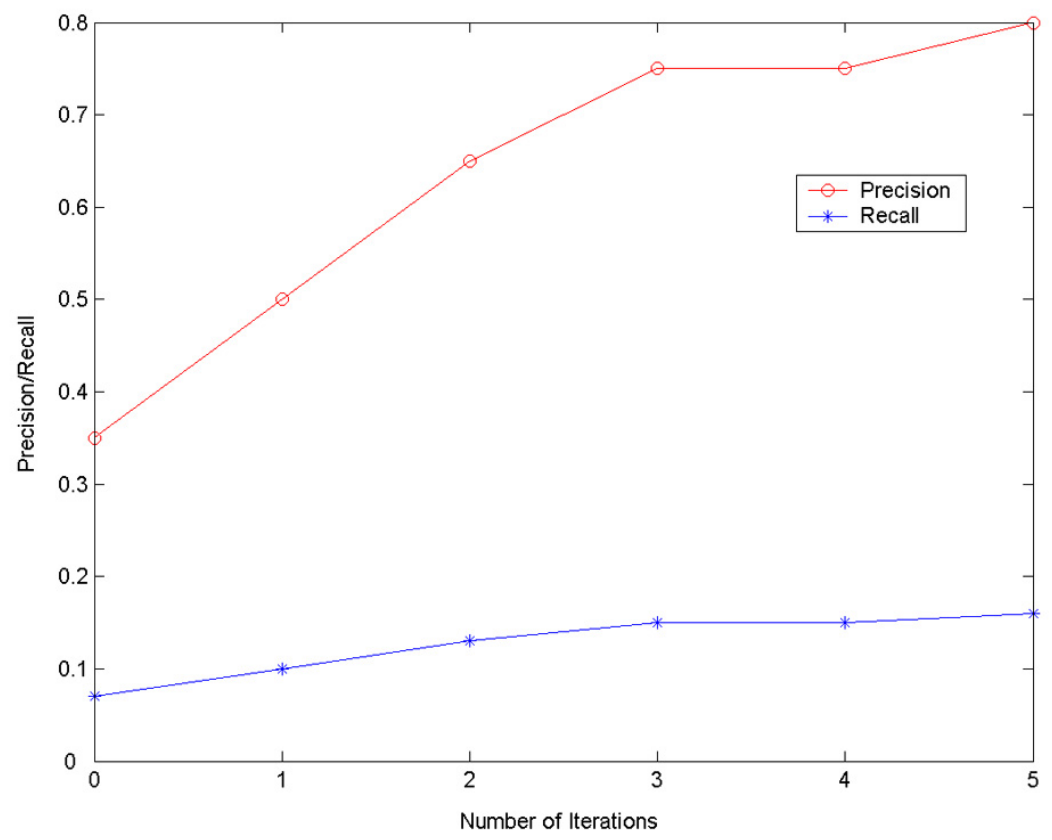

Fig. (2). Precision and recall curves with different iterations.

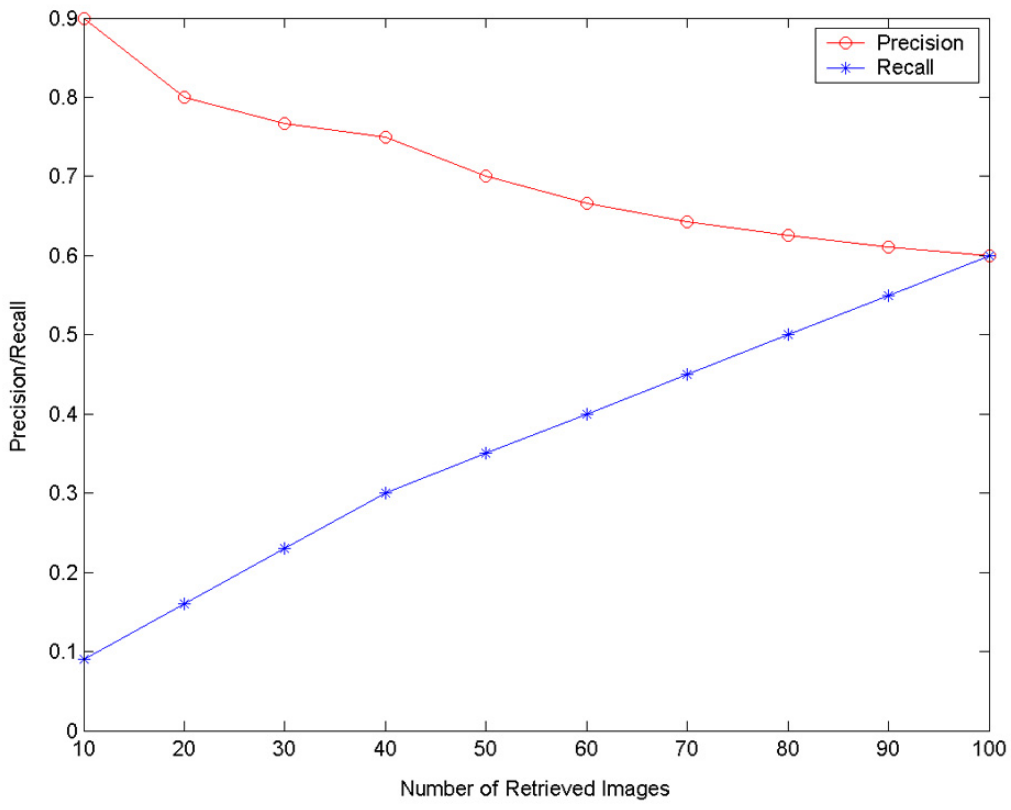

Fig. (3). Precision and recall curves with different returned images number.

The last performance measurements are the averaged value over 10 queries. We use the precision and recall curve as the performance measurements.

The contrast curves of precision and recall with different feedback stage are given in Fig. (2). The curves illustrate that the two measurements gradually increase during user feedback. The precision values are 0.5 at first feedback and 0.8 at fifth feedback, respectively. The recall values are 0.1 at first feedback and 0.16 at fifth feedback, respectively. In the initial iteration stage, the retrieval performance improves quickly, but slowly in the subsequent iteration stage. This means that the approach converges quickly to retrieve image satisfying the user's need.

In the case of various numbers of returned images, the contrast curves of precision and recall are illustrated in Fig. (3). The curves show that the precision decreases with the increase of the returned images number, but the recall increases. Because the number of returned images increases, 
more and more negative images are retrieved and returned to the user for feedback, which will affect the precision. When the number of retrieved images reaches 100 , which is the number of total positive images in the database, the two measurements are equal.

\section{CONCLUSION}

A new relevance feedback technique for semantic image retrieval based on the self-growing radial basis function neural network is proposed in the paper. The approach can adaptively construct SGRBF neural network based on the users' feedbacks. Thus, hidden nodes of the SGRBF neural network can represent the distribution of the users' perceptual in the low-level feature space and bridge the semantic gap between low-level feature and high-level concept of the image content. Experimental results show that the proposed method have greatly improved the performance of semantic image retrieval.

\section{ABOUT THE AUTHORS}

First Author Li Guizhi, Associate Professor of Beijing Information and Science and Technology University, Ph.D. The author's major is Computer Applications, and the research fields include machine learning, image retrieval, artificial intelligence, etc. More than 10 papers have been published and received by EI.

Second Author Huang Hongbo, Lecturer of Beijing Information and Science and Technology University, master degree in Computer Applications, studying for $\mathrm{PhDs}$ in Beijing University of Science and Technology. The author's research fields include image processing, machine vision, etc.

\section{CONFLICT OF INTEREST}

The author confirms that this article content has no conflict of interest.

\section{ACKNOWLEDGEMENTS}

This work was supported by a grant from Funding Project for Academic Human Resources Development in Institutions of Higher Learning under the Jurisdiction of Beijing Municipality. (No.PHR201108261)

\section{REFERENCES}

[1] Y.Rui, T. S. Huang, M.Ortega, S.Mehrotra, "Relevance Feedback: A Power Tool for Interactive Content-Base Image Retrieval”, IEEE Transactions on Circuits and Video Technology, vol. 8, no.5, pp. 644-655, 1998 .

[2] D.H. Kim, C.W. Chung, K. Barnard, "Relevance Feedback Using Adaptive Clustering for Image Similarity Retrieval", The Journal of Systems and Software, vol.78, pp. 9-23,2005.

[3] Z.H. Zhou , K. J. Chen, Y. Jiang, "Exploiting Unlabeled Data in Content-Based Image Retrieval",In Proceedings of the 15th European Conference on Machine Learning, 2004, pp.525-536.

[4] L. Wang, K.L. Chan, Z. Zhang, "Bootstrapping SVM Active Learning by Incorporating Unlabelled images for Image Retrieval", Proc. IEEE Int. Conf. Computer Vision and Pattern Recognition, 2003, pp. 629-634.

[5] G. Giacinto, "A Nearest-Neighbor Approach to Relevance Feedback in Content Based Image Retrieval", In Proceedings of the 6th ACM international conference on Image and video retrieval, 2007, pp.456-463.

[6] J. W. Kwaka, N. Chob, “ Relevance feedback in content-based image retrieval system by selective region growing in the feature space", Signal Processing: Image Communication, vol.18, pp.787799, 2003.

[7] K. Chandramouli, T. Kliegr, J. Nemrava, V.Svatek and E. Izquierdo, "Query Refinement and User Relevance Feedback for Contextualized Image Retrieval", In Proceeding of Visual Information Engineering, 2008, pp. 453-458.

[8] M.M. Rahman, P. Bhattacharya, B.C. Desai, "A Framework for Medical Image Retrieval Using Machine Learning and Statistical Similarity Matching Techniques With Relevance Feedback”, IEEE Transactions on Information Technology in Biomedicine, vol.11, No.1, pp.58-69, 2007.

[9] Y. Ishikawa, R. Subramanya, "MindReader: Query Database Through Multiple Examples", Proc. Of Int. Conf. on Very Large Data Bases, New York, USA, 1998, pp.651-675.

[10] P. Muneesawang, L. Guan, "Automatic Machine Interactions for Content-Based Image Retrieval Using a Self-Organizing Tree Map Architecture", IEEE Trans. on Neural Networks, vol. 13, no. 4, pp. 821-834,2002.

[11] R.J. Liu, Y.H. Wang, T. Baba, D. Masumoto, S. Nagata, "SVMBased Active Feedback in Image Retrieval Using Clustering and Unlabeled Data”, Pattern Recognition, vol.41, pp.2645-2655,2008.

[12] C. L. Lin, S.T. Hsieh, T.Y. Sun, C.C. Liu, "Cluster Distance Factor Searching by Particle Swarm Optimization for Self-Growing Radial Basis Function Neural Network", International Joint Conference on Neural Networks, 2006, pp.4825-4830.

[13] Y. X. Chen, J.Z. Wang, R. Krovetz. "CLUE: Cluster-Based Retrieval of Images by Unsupervised Learning", IEEE Transactions on Image Processing, vol.14, no.8, pp.1187-1201, 2005.

[14] I. Lee, L. Guan, "Content-based image retrieval with automated relevance feedback over distributed peer-to-peer network", IEEE International Symposium on Circuits and Systems, 2004,pp. 5-8.

(C) Guizhi and Hongbo; Licensee Bentham Open.

This is an open access article licensed under the terms of the Creative Commons Attribution Non-Commercial License (http://creativecommons.org/licenses/by$\mathrm{nc} / 3.0 /$ ) which permits unrestricted, non-commercial use, distribution and reproduction in any medium, provided the work is properly cited. 